\title{
Leadership and Cultural Issues: Evaluation and Measurement in the Context of Software Development Projects
}

\author{
Khaled Hamdan, Peter Smith, and Valentina Plekhanova
}

\begin{abstract}
The success of a software project requires the work of highly capable and motivated individuals as well as a strong and capable leader. Yet, it has been repeatedly demonstrated that lack of quality leadership in a project is often a cause of that project's ultimate failure. Selecting the most suitable individual to lead a project is not an easy task. In this research we present a new method for selecting a project manager, by formally modeling leadership and cultural characteristics. The characteristics sought by the organisation and of each candidate manager are modeled using an approach known as profile theory. We show how to apply the methodology within an organisational context.
\end{abstract}

Index Terms-Culture and leadership characteristics, profile theory, project management, quantitative assessment.

\section{INTRODUCTION}

Most models of leadership style (e.g. Leadership Traits; Leadership Grid; Contingency approaches; Path-Goal theory) are descriptive [1], [2]. They were developed in the context of western culture and for traditional (e.g. manufacturing, construction) organisations. The value of this research is that the proposed method attempts to model different leadership styles and cultures. The proposed work offers a holistic, integrated and normative approach for the selection of a leader/project manager.

A survey of software development projects within government departments in the United Arab Emirates (UAE) was undertaken by the authors [3]. We analyzed data from more than 20 government organisations in the UAE. The process of collecting information was conducted in three stages, each using a different survey instrument. The survey asked questions as to whether, and how, cultural and leadership factors have an impact on the accuracy of software effort and cost estimation. The survey results indicated that respondents in UAE recognise the significance of cultural aspects when carrying out effort estimation. Our work also demonstrated that community, organisational and team cultures are intertwined. Individuals are unable to escape their culture and background; they all affect each other. The final version of the survey involved the collection of data on seven parameters: Organisations Line of Business, Application Type, Organisation Type, Organisational Culture, Project

Manuscript received January 16, 2012; revised February 25, 2012.

K. Hamdan is with UAE University, Al Ain, UAE (e-mail: khamdan@uaeu.ac.ae).

P. Smith and V. Plekhanova are with Sunderland University, UK (email: Peter.Smith@sunderland.ac.uk,
Leadership, Project Technical Environment and Year of Project Completion [3], [4], [5].

Numerous studies [3], [6], [7] have tried to identify the impact of cultural traits and leadership characteristics, on the total cost [6] of a software development project. Researchers have repeatedly shown that lack of leadership within a project is often the cause of ultimate failure [8]. The leader of an organisation has an essential role to play in setting the vision for their organisation. To that end, the authors undertook a further study that aimed to identify the importance of project leadership factors in estimating software development cost in the Arabian Gulf States. In that study a survey of software development projects within government departments was undertaken. Analysis highlighted several factors which affect software cost estimation; these can however, be grouped under the broad headings of leadership and cultural characteristics.

From this work [3], [4], the authors concluded that leadership and cultural characteristics are very important when selecting a project manager. The goal of this paper is to propose a methodology by which profile factors can be analyzed and ranked, and the most appropriate manager selected. To achieve this goal, organisational characteristics, and those of candidates are modeled.

Consideration of how best to model managerial practices has been addressed [9]. In this paper, the authors use a formalism known as profile theory [10]. We define a leadership profile that contains common data and constraints. As a result, we remove the pitfall of data redundancy. In our model, a value for the profile is computed, based on leadership and cultural factors. After presenting the model, we show how to apply it using leadership characteristics as an example. However, the method could be extended to include other aspects of project management such as technical characteristics [11].

The paper commences as follows. Section II identifies key leadership and cultural factors. Section III discusses leadership characteristics. Section IV discusses exploratory of the Data analysis. Section V presents the model. Section VI presents outlines the selection algorithm, and Section VII demonstrates its use. Finally, Section VIII provides a conclusion for this paper.

\section{IDENTIFYING THE KEY CULTURAL AND LEADERSHIP FACTORS}

The measurement of organisational culture and leadership in software project development and in cost estimation is complex. Fig. 1 illustrates the factors and structure that affect team culture and leadership. This 
diagram has been derived by the authors from analysis of the data obtained from our survey work.

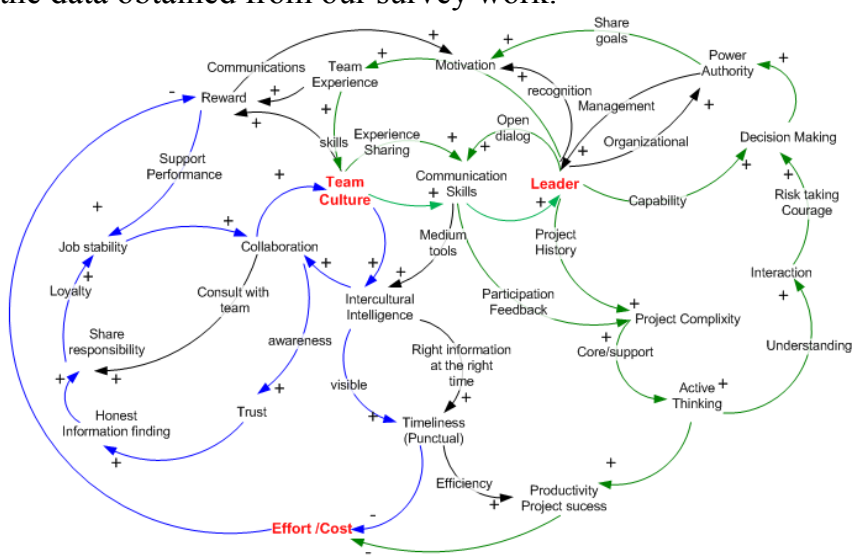

Fig. 1. Factors and their relationships affecting team culture and leadership [4]

There are two main positive feedback loops. The first feedback loop starts at the node labelled "Team Culture". Good team culture improves punctuality and timeliness which, in turn, reduce effort and cost. Clear estimates and successful projects will improve reward systems, resulting in job stability. Also, as team members' subjectivity is avoided, collaboration will increase. This feeds back into the team culture which completes the loop. Optimizing the flow in this structure will result in an ideal bond between leadership and culture; consequently, the effectiveness of this team is maximized.

The second feedback loop starts at the node labelled "Leader". This represents good leadership based on recall ability of historical data and active thinking. This increases productivity, resulting in lower cost and effort which, in turn, gives better rewards and support to the team. Leadership increases collaboration and team culture through sharing experiences and communication skills. Again this feeds back into leadership and completes the loop.

\section{LEADERSHIP AND CULTURAL CHARACTERISTICS}

Based on the survey of software firms, Hamdan [4] identified six attributes that characterise leadership in the context of a software project. These have an impact on the cost of software project execution and can be used to discern the performance of one project manager from another. The characteristics are:

- Interaction and relationship

- Decision-making

- Ability to motivate

- Understanding project culture

- Active thinking

- Communication skills

Hamdan [4] also analysed the literature to identify the most relevant cultural characteristics. This work identified the following seven cultural characteristics:

- Timeliness

- Collaboration

- Job stability

- Intercultural intelligence

- Reward mechanism
- Communication

- $\quad$ Team experience

Leadership and cultural characteristics were measured using multiple features. Leadership was measured by six items and each item was assessed by four sub-items. Cultural characteristics were measured by seven items and each item was also assessed by four sub-items (See Appendix A). The cultural and leadership characteristics sub-items were then measured using a 9 point ordinal scale. This was chosen to allow a broad analysis of the response. The respondents were directed to assign a value ranging from the lowest score of 1 to the highest score of 9 . The scores of the sub-items were then averaged separately for each item, resulting in a single item score. These factors use a broad 9 point scale (exceptionally high $=9$, very high $=8$, high $=7$, fairly high $=6$, sufficient $=5$, average $=4$, low $=3$, unsatisfactory $=2$, exceptionally low $=1$ ).

In an attempt to identify attributes characterizing culture and leadership, administrators, managers and project leaders were surveyed. The literature review has shown that leadership and culture go hand in hand. Both are important factors in the way an organisation functions.

Each of the leadership and cultural characteristics were then weighted with respect to its significance and influence to a software project. The experience of project resource was rated against each characteristic on a scale from 1 to 9 . In addition, the competence of each candidate was assessed (1=Junior, 2=Senior, 3=Principal).

The weights for leadership and cultural compatibility are computed by the number of required and number of available factors. The measured capabilities and compatibilities are used to determine how close the candidate's case is to the required case or to an average case. The decision may be based on closest weight.

In order to obtain a ranking for leadership, each characteristic was weighted. These weights were obtained empirically from surveys and interviews with managers and employees from relevant organisations [4].

\section{EXPLORATORY ANALYSIS OF THE DATA}

The data analysis is important to identify patterns and relationships which help form judgments about the attributes being measured. In order to measure these crucial human characteristics objectively and accurately, reliability is needed throughout software development rather than at the end of the process. These measurements may prove valuable in the building of effort prediction models.

Several statistical techniques were used to analyse the data [4]. Descriptive and graphical methods were used to explore and describe the values of attributes, according to the type of project and organisation. A student t-test and a one-way analysis of variance (ANOVA) were used to determine significant differences in project attributes; according to the type of project and organisation. Associations between attributes were assessed using Pearson's correlation for quantitative data and the Chisquare test of independence for qualitative data. Regression analysis was used to develop an equation for explaining the relationship between actual effort and software project 
attributes. The Kolmogorov-Smirnov test was used to assess the normality of data. Descriptive statistics for the aforementioned leadership and culture variables was obtained [7].

TABLE I: CORRELATION BETWEEN CULTURE AND LEADERSHIP CHARACTERISTICS

\begin{tabular}{|c|c|c|c|c|c|c|c|c|}
\hline & & Timeliness & $\begin{array}{c}\text { Collaboration } \\
\text { (Interpersonal } \\
\text { Relation) }\end{array}$ & $\begin{array}{c}\text { Job } \\
\text { Stability }\end{array}$ & $\begin{array}{l}\text { Intercultural } \\
\text { Intelligence }\end{array}$ & $\begin{array}{c}\text { Reward } \\
\text { Mechanism }\end{array}$ & Communication & $\begin{array}{c}\text { Team } \\
\text { Experience }\end{array}$ \\
\hline \multirow{2}{*}{$\begin{array}{l}\text { Interaction and } \\
\text { Relationships }\end{array}$} & Pearson Correlation & $.692^{\star \star}$ & $.690^{\star *}$ & $.738^{* *}$ & $.571^{\star \star}$ & $.458^{* *}$ & $.585^{\star \star}$ & .165 \\
\hline & Sig. (2-tailed) & .000 & .000 & .000 & .000 & .004 & .000 & .322 \\
\hline \multirow[t]{2}{*}{ Decision-Making } & Pearson Correlation & $.677^{\star \star}$ & $.849^{* \star}$ & $.628^{* *}$ & $.529 * *$ & $.571^{* \star}$ & $.706^{* *}$ & $.338^{*}$ \\
\hline & Sig. (2-tailed) & .000 & .000 & .000 & .001 & .000 & .000 & .038 \\
\hline \multirow[t]{2}{*}{ Ability to Motivate } & Pearson Correlation & $.598^{* *}$ & $.790^{* *}$ & $.610^{* *}$ & $.472^{* *}$ & $.666^{* *}$ & $.487^{* *}$ & .092 \\
\hline & Sig. (2-tailed) & .000 & .000 & .000 & .003 & .000 & .002 & .583 \\
\hline \multirow{2}{*}{$\begin{array}{l}\text { Understanding } \\
\text { Organisation Culture }\end{array}$} & Pearson Correlation & $.714^{\star \star}$ & $.773^{* *}$ & $.741^{* *}$ & $.644^{* *}$ & $.690^{\star *}$ & $.449^{* *}$ & .132 \\
\hline & Sig. (2-tailed) & .000 & .000 & .000 & .000 & .000 & .005 & .430 \\
\hline \multirow[t]{2}{*}{ Active Thinking } & Pearson Correlation & $.609^{\star \star}$ & $.750^{\star \star}$ & $.558^{* *}$ & $.398^{* *}$ & $.538^{\star \star}$ & $.591^{* *}$ & .242 \\
\hline & Sig. (2-tailed) & .000 & .000 & .000 & .013 & .000 & .000 & .144 \\
\hline \multirow{2}{*}{$\begin{array}{l}\text { Communication } \\
\text { skills }\end{array}$} & Pearson Correlation & $.580^{\star \star}$ & $.776^{\star \star}$ & $.501^{\star \star}$ & $.469^{\star *}$ & $.611^{\star \star}$ & $.701^{\star \star}$ & $.345^{\star}$ \\
\hline & Sig. (2-tailed) & .000 & .000 & .001 & .003 & .000 & .000 & .034 \\
\hline
\end{tabular}

${ }^{* *}$. Correlation is significant at the 0.01 level (2-tailed). ${ }^{*}$.Correlation is significant at the 0.05 level (2-tailed).

Table I shows a strong correlation between leadership and cultural characteristics. The only exception is 'Team Experience' which seems to correlate only with 'Reward Mechanism' and 'Communications'. Some leadership and cultural characteristics appear to be more important than others. These characteristics were believed by the respondents to be significant attributes in most cases. This is probably due to the fact that these are innate attributes which are part of the individuals' characters. These have been shaped by interaction with others and by life experiences.

\section{MODELLING LEADERSHIP ASPECTS}

It is crucial to identify the common characteristics that add to a project success and to be used as factors or dimensions. Thus, an important decision is how to select an suitable leader for a given project. A proposed process consists of defining a leadership factors tree. This tree is derived from the feedback of factors and their relationships captured in the common causal diagram. The factors are each given weights, based on their importance. In order to identify leadership aspects that could impact upon software team performance and effort estimation, the authors undertook an extensive literature review [4]. This considered key generic aspects of leadership that contribute to cost estimation. These common aspects were found to be [4], [12]:

- Team culture

- Leadership style

- Complexity of project

- Organisational type

- Qualification

Team culture is assessed based on experience level pertaining to the task under investigation, the level of confidence and hard work [12]. The organisational types are defined as [2], [12]:

- Project oriented

- Functional

- Matrix

Project complexity is measured by the type of the application and entities [4], [13]. Project complexity is categorised into two areas:
- Core systems

- $\quad$ Supporting systems.

Core systems exist to achieve the core mission of organisations and to satisfy their core purpose. Examples of core systems are: Fire alarm systems used by civil defense departments, Flight information systems used by civil aviation departments, Traffic light management systems in police and traffic departments. Supporting systems support the internal services of an organisation. Examples of supporting systems are: Human resources and payrolls, financial systems, Document management systems.

Leadership skill is defined by communication channels and the ability to exchange information and ideas from one to another [11]. Attributes such as power and traits describe the leadership style [8], [9].

The leadership aspects (e.g. leadership style, team culture, organisational type, communication skills, project complexity) that were identified from the literature review and the interviews of IT managers/leaders are shown in the form of a tree in Fig. 2 [4].

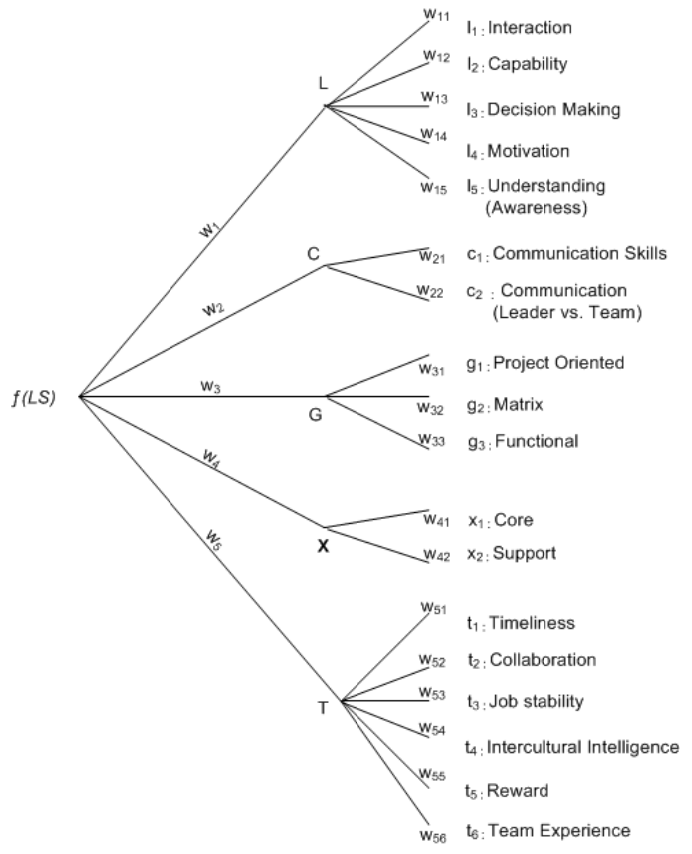

Fig. 2. Leadership factors tree 
L: Leadership characteristics

$C$ : Communication skills

$G$ : Organisational type

$X$ : Project complexity

$T$ : Team culture

Each leadership aspect could be defined by a set of factors that were identified and discussed in [4]. It is difficult to analyse all leadership factors that could affect the cost estimation models. Leadership characteristics and other variables involve quantitative measures of capability. The completeness property is important for the identification of the essential profile factors and it is equally important for these to be incorporated and combined into the profile description. Consideration of multiple leadership aspects can be accommodated within
Profile Theory [9]. Profile Theory offers an integrated approach to modelling complex systems where capability and compatibility are critical factors.

Qualitative aspects are defined by the qualitative values of multi-dimensional leadership aspects and relevant factors; and a (preference/importance) weight is allocated to each factor. Leadership factors have multiple properties (see Table II) and they are presented by a profile superposition [10]. The most important factors have the highest weight/priority weights. An example of quantitative description of leadership aspects is that each has a number of factors. Actual values were recorded and given similar weight in ad hoc cases. A total required weight was computed to measure leadership characteristics.

TABLE II: QUANTITATIVE DESCRIPTION OF LEADERSHIP ASPECTS

\begin{tabular}{|c|c|c|c|c|c|c|c|c|}
\hline Description & Aspects & $\begin{array}{c}\text { No of Factors } \\
\text { (n) }\end{array}$ & $\begin{array}{l}\text { Max Scale } \\
\text { (s) }\end{array}$ & Factors & $\begin{array}{l}\text { Actual Value } \\
\text { (z) }\end{array}$ & $\begin{array}{l}\text { Max Factor } \\
\text { Weight (w) }\end{array}$ & $\begin{array}{l}\text { Weight Aspect } \\
\text { (Q) }\end{array}$ & Z_norm \\
\hline \multirow{5}{*}{ Leadership } & \multirow{5}{*}{$\mathrm{L}$} & \multirow{5}{*}{5} & \multirow{5}{*}{9} & 11 & 7.1 & \multirow{5}{*}{0.20} & \multirow{5}{*}{0.040} & 0.032 \\
\hline & & & & 12 & 7.0 & & & 0.031 \\
\hline & & & & 13 & 6.6 & & & 0.029 \\
\hline & & & & 14 & 6.8 & & & 0.030 \\
\hline & & & & 15 & 7.2 & & & 0.032 \\
\hline \multirow{8}{*}{ Team Culture } & \multirow{7}{*}{ T } & \multirow{7}{*}{6} & \multirow{7}{*}{9} & & & \multirow{7}{*}{0.2} & $w(L)$ & 0.15 \\
\hline & & & & t1 & 6.7 & & \multirow{6}{*}{0.033} & 0.025 \\
\hline & & & & t2 & 6.9 & & & 0.026 \\
\hline & & & & t3 & 6.8 & & & 0.025 \\
\hline & & & & $\mathrm{t} 4$ & 7.1 & & & 0.026 \\
\hline & & & & t5 & 6.5 & & & 0.024 \\
\hline & & & & t6 & 6.8 & & & 0.025 \\
\hline & \multirow{4}{*}{ G } & & & & & & $w(T)$ & 0.15 \\
\hline \multirow{4}{*}{ Organisational Type } & & \multirow{3}{*}{1} & \multirow{3}{*}{3} & Functional & 0 & \multirow{3}{*}{0.2} & \multirow{3}{*}{$k=0|1 / 2| 1$} & - \\
\hline & & & & Matrix & 0.5 & & & - \\
\hline & & & & Pri. Oriented & 1 & & & 0.200 \\
\hline & & & & & & & $w(G)$ & 0.20 \\
\hline \multirow{3}{*}{ Communication Skills } & \multirow{3}{*}{ c } & \multirow{3}{*}{2} & \multirow{2}{*}{9} & Leader C & 7.1 & \multirow{2}{*}{0.2} & \multirow{2}{*}{0.10} & 0.079 \\
\hline & & & & Team C & 7.1 & & & 0.079 \\
\hline & & & & & & & $w(\mathbf{C})$ & 0.13 \\
\hline \multirow{4}{*}{ Project Complexity } & \multirow{3}{*}{$\mathrm{x}$} & \multirow{2}{*}{1} & \multirow{2}{*}{2} & Support & 0 & \multirow{2}{*}{0.2} & \multirow{2}{*}{$k=0 \mid 1$} & 0.100 \\
\hline & & & & Core & 1 & & & - \\
\hline & & & & & & & $w(X)$ & 0.10 \\
\hline & & & & & & & Total required weight $(\mathrm{Z})$ & 0.67 \\
\hline
\end{tabular}

The factors that represent leadership $(L S)$, are introduced by the following profile superposition:

$$
\begin{gathered}
f(L S)=\left\{<\varepsilon_{1}, L, \omega_{1}>,<\varepsilon_{1}, T, \omega_{1}>,<\varepsilon_{1}, G, \omega_{1}>\right. \\
\left.<\varepsilon_{1}, C, \omega_{1}>,<\varepsilon_{1}, X, \omega_{1}>\right\}
\end{gathered}
$$

where

$\varepsilon_{i}$ : Factor existence such as $\varepsilon=1$, non existence $\varepsilon=0$; where $\varepsilon_{i}$ is $\varepsilon_{1}, \varepsilon_{2}, \varepsilon_{3}, \varepsilon_{4}$, and $\varepsilon_{5}$ are the factor existence for leader characteristics, i.e. information/data about the ith factor (i.e. team culture, organisational type, communications skill, and complexity) is available $\left(\varepsilon_{i}=1\right)$ or not $\left(\varepsilon_{i}=0\right)$,

$\omega_{i}$ : the $i$ th factor (preference/importance) weight; where $\omega_{1}$, $\omega_{2}, \omega_{3}, \omega_{4}$, and $\omega_{5}$, are the weights for leadership characteristics, team culture, organisational type, communications skill, and complexity respectively. For example a leader profile may be defined by five parameters to measure the leadership factor.

Leadership aspects $(L)$ are defined by the following profile superposition:

$$
L=\left\{\left(<\varepsilon_{i}, l_{i}, \omega_{i}>\right), i=\overline{1, n}\right\}, n=5 \text {, i.e. }
$$

$L=\left\{\left(<\varepsilon_{i}, l_{i}, \omega_{i}>\right)\right\}$

$$
=\left\{\begin{array}{rr}
l 1, & \text { is } \text { Interaction and Relationship } \\
l 2, & \text { is Decision - Making } \\
l 3, & \text { is Ability to motivate } \\
l 4, & \text { is Understand Project Culture } \\
l 5, & \text { is Active Thinking }
\end{array}\right.
$$

For example, leadership aspects introduced in the Table II define the following leadership characteristics profile:

$$
\left.\mathrm{L}=\left\{<1,7.1, \frac{1}{5}\right\rangle,<1,7.0, \frac{1}{5}>, \ldots\right\} .
$$

Let us describe the Team Culture profile $(T)$ by the following 6 factors such the experience of the team, intercultural capability and interaction of the members of the team.

$$
T=\left\{\left(<\varepsilon_{i}, t_{i}, \omega_{i}>\right), i=\overline{1, n}\right\}, n=6 \text {, i.e }
$$

$T=\left\{\left(<\varepsilon_{i}, t_{i}, \omega_{i}>\right)\right\}=\left\{\begin{array}{lr}t 1, & \text { is Timeliness } \\ t 2, & \text { is Collaboration } \\ t 3, & \text { is Job Stability } \\ t 4, & \text { is Intercultural Intelligence } \\ t 5, & \text { is Reward Mechanism } \\ t 6 & \text { is Team Experience }\end{array}\right.$ 
The organisational type $(G)$ profile may be defined by 3 parameters

$$
\begin{aligned}
& G=\{(\left.\left.<\varepsilon_{i}, g_{i}, \omega_{i}>\right), i=\overline{1, n}\right\}, n=3, \text { i.e } \\
& G=\left\{\left(<\varepsilon_{i}, g_{i}, \omega_{i}>\right)\right\} \\
&=\left\{\begin{array}{l}
g 1, \quad \text { is Functional (less authority) } \\
g 2, \text { is Matrix (share similar authority) } \\
g 3 \text { is Project Oriented (high authority) }
\end{array}\right.
\end{aligned}
$$

The communication skills profile may be defined as:

$$
\begin{gathered}
C=\left\{\left(<\varepsilon_{i}, c_{i}, \omega_{i}>\right), i=\overline{1, n}\right\}, n=2 \text {, i.e } \\
C=\left\{\left(<\varepsilon_{i}, c_{i}, \omega_{i}>\right)\right\}= \begin{cases}c 1, & \text { is Team communication } \\
c 2, & \text { is Leadership communication }\end{cases}
\end{gathered}
$$

The project complexity $(X)$ profile may be defined by 2 parameters

$$
\begin{array}{r}
X=\left\{\left(<\varepsilon_{i}, x_{i}, \omega_{i}>\right), i=\overline{1, n}\right\}, n=2, \text { i.e } \\
X=\left\{\left(<\varepsilon_{i}, x_{i}, \omega_{i}>\right)\right\}=\left\{\begin{array}{lr}
x 1, & \text { is Support systems } \\
x 2, & \text { is Core systems }
\end{array}\right.
\end{array}
$$

For the purpose of illustration we allocate equal weights to each leadership aspect. Therefore, $\omega_{\mathrm{i}}=1 / \mathrm{n}, 0 \leq \omega_{\mathrm{i}} \leq$ $1 / n$ where $n$ is a number of factors that are used for description of leadership aspects; $\omega_{i}$ is the ith factor (preference/ importance) weight.

Now let us define by:

$Z_{i}$ : actual value for each factor in leadership aspect description

$Q_{i}$ : weight of each leadership aspect

$S_{i}$ : maximum scale value for each leadership aspect

$n_{i}$ : number of factors of leadership aspects

We define a weight $Q_{i}$ for each factor by $Q_{i}=\frac{\omega_{i}}{n_{i}}$, for example (see Table I): each leader characteristic weight is defined as $\frac{0.2}{5}=0.04$, where 5 is number of factors for leadership description. The weight for each factor is based on an actual value $\left(Z_{i}\right)$ multiplied by the weight of each factor $\left(\mathrm{Q}_{\mathrm{i}}\right)$ and divided by the maximum scale value $\left(S_{i}\right)$.

Let us define normalized actual value for each factor by the following formula:

$$
Z_{\text {norm }(i)}=\frac{Z_{i} \times Q_{i}}{S_{i}}
$$

We need to normalize these on a scale of $[0,1]$. Normalization here is needed in order to avert undesired influence of one characteristic to the others. We apply range normalization in order to obtain the new value. In this particular case, we take into account a contribution of each characteristic (of the profile superposition) to the leadership profile description.

$$
Z_{i}=\sum_{i=1}^{n} \frac{Z_{\text {norm }(i)} \times Q_{i}}{S_{i}}
$$

where $n$ is a number of aspects that are used for leadership description.

For example, let use consider Table I. The total value of normalized actual values for all factors that are used for description of leadership aspect is:

$$
\begin{gathered}
Z_{i}=\sum_{i=1}^{n} \frac{Z_{\text {norm }(i)} \bullet Q_{i}}{S_{i}}=0.154+0.151+0.200+ \\
0.128+0.100=0.67
\end{gathered}
$$

For this case, by adding all sub factors for leadership will give a ratio which can be compared with other leadership to see which profile has more weight on the project success.

An aspect profile capability $\left(V_{i}\right)$ for leadership is defined as a sum of all factor capabilities [5]:

$$
V_{i}=\sum_{j=1}^{m_{i}} \omega_{j}\left(\frac{\varepsilon_{j}}{\varepsilon_{j}^{(())}}\right)\left(\frac{v_{j}}{v_{j}^{(())}}\right)^{2}
$$

where for the $j$ th factor:

$\varepsilon_{j} \quad$ : factor existence such as $\varepsilon=1$, non existence $\varepsilon=0$

$\varepsilon_{j}^{(0)}$ : required experience, $\varepsilon_{j}^{(())} \neq 0$

$v_{j}$ : existing level of factors

$v_{j}^{(0)}$ : required level of factors, $v_{j}^{(0)} \neq 0$

$\omega_{j}:$ the $j$ th factor (preference/importance) weight

$m_{i}$ : number of factors that are used for the description of leadership aspects

In order to determine compatibility of the availble leadership with the required one we use the following compatibility measure [9], [10]:

$$
W_{i}=\prod_{j=1}^{m_{j}}\left(\frac{\varepsilon_{j}}{\varepsilon_{j}^{(())}}\right)\left(\frac{v_{j}}{v_{j}^{(0)}}\right)^{2}
$$

where $W_{i}$ is the leadrship aspect compatibility.

Let us consider the following illustrative example using Table III. Leadership aspects of project manager are interactions and relations, decision-making, ability to motivate team members, ability to understand organisational culture, and active thinking (see Table II). Each project managers PM (I), PM (II) and PM (III) leadership available capability and compatibility are compared with the required leadership capability and compatibility. That is, we need to compute leadership available capability and compatibility for each project manager.

The leadership aspect is described by 5 factors. The required level of the leadership is advanced/high $=3$. The weight is equal priority for all factors, i.e. $\omega_{j}=1 / 5$. For example, the leadership profile capability for PM (I) is:

$$
V_{(l 1)}=0.20\left(\frac{7.1}{9}\right)\left(\frac{3}{3}\right)^{2}=0.158
$$

where the levels scale is: 1 for low, 2 for nominal and 3 for high/advanced. The total capability $V_{(l)}$ is the sum of all $V_{(l i)}$, where $i$ is the number for factors. The length is the number of factors available or satisfies required profile.

Table III introduced a summery of leadership capability for three project managers. The capabilities for PM (I), PM (II), and PM (III) are computed as 0.771, 0.572, and 0.291 
respectively. PM (I) shows higher capability profile based on selected values. The compatibility shows higer for PM(I) than other candidates.

The compatibilities weight were computed using all leadership aspects. The profile for PM(I) shows 0.272 and factors weight available capability is 0.340 . The model described puts the foundation for devising a simple algorithm for selecting the most suitable project manager profile factors that matches the announced organisation job position. Regarding the leadership aspects for capability and compatibility values, all factors have equal weights 0.2 . Based on the aspects capability and compatibility of these alternative project managers, a suitable candidate is allocated. A similar approach was implemented with culture and project resource profiles.

TABLE III: LEADERSHIP ASPECTS FOR CAPABILITY AND COMPATIBILITY VALUES

\begin{tabular}{|c|c|c|c|c|c|c|c|c|c|c|c|c|}
\hline Leadership Aspect & & quirec & & & M (I) & & & $M(I I)$ & & & $M(I I I)$ & \\
\hline & attrib & level & weight & attrib & level & weight & attrib & level & weight & attrib & level & weight \\
\hline Interactions and Relations & 9 & 3 & 0.20 & 7.1 & 3 & 0.20 & 7.5 & 3 & 0.20 & 6.0 & 3.0 & 0.2 \\
\hline Decision-Making & 9 & 3 & 0.20 & 7.0 & 3 & 0.20 & 6.2 & 2 & 0.20 & 5.0 & 1.0 & 0.2 \\
\hline Motivate team members & 9 & 3 & 0.20 & 6.6 & 3 & 0.20 & 6.3 & 3 & 0.20 & 6.0 & 3.0 & 0.2 \\
\hline Understand organisational culture & 9 & 3 & 0.20 & 6.8 & 3 & 0.20 & 6.0 & 2 & 0.20 & 5.0 & 1.0 & 0.2 \\
\hline Active Thinking & 9 & 3 & 0.20 & 7.2 & 3 & 0.20 & 6.5 & 3 & 0.20 & 0.0 & 0.0 & 0.0 \\
\hline Others (Communication Skills) & 0.0 & 0.0 & 0.00 & 7.1 & 3 & 0.20 & 0.0 & 0.0 & 0.0 & 0.0 & 0.0 & 0.0 \\
\hline Leadership Capability & $v(l)$ & $w(l)$ & & $v(l 1)$ & $w(l 1)$ & & $v(12)$ & $w(12)$ & & $v(l 3)$ & $w(l 3)$ & \\
\hline$v(l 1)$ & 0.20 & 1.00 & & 0.158 & 0.789 & & 0.167 & 0.833 & & 0.133 & 0.667 & \\
\hline$v(l 2)$ & 0.20 & 1.00 & & 0.156 & 0.778 & & 0.061 & 0.306 & & 0.012 & 0.062 & \\
\hline$v(l 3)$ & 0.20 & 1.00 & & 0.147 & 0.733 & & 0.140 & 0.700 & & 0.133 & 0.667 & \\
\hline$v(l 4)$ & 0.20 & 1.00 & & 0.151 & 0.756 & & 0.059 & 0.296 & & 0.012 & 0.062 & \\
\hline$v(15)$ & 0.20 & 1.00 & & 0.160 & 0.800 & & 0.144 & 0.722 & & 0.000 & 0.000 & \\
\hline$v(l)$ & 1.000 & & & 0.771 & & & 0.572 & & & 0.291 & & \\
\hline Length & 5 & & & 5 & & & 5 & & & 4 & & \\
\hline$p(l)$-compatibility length & 1.000 & & & 1.000 & & & 1.000 & & & 0.800 & & \\
\hline$w(l)$ - compatiblity weight & 1.000 & & & 0.272 & & & 0.038 & & & 0.000 & & \\
\hline$w(l(1 / 5))$ compatiblity weight & 1.000 & & & 0.340 & & & 0.053 & & & 0.002 & & \\
\hline
\end{tabular}

The compatibility length $\left(p_{l}\right)$ is ratio of available factors over required factors. For example, the $p_{l}$ for PM (I) is: $5 / 5$

$$
p_{l}=\frac{\text { Available factors }}{\text { Required factors }} \leq 1
$$

The compatibility weight $\left(\omega_{l}\right)$ is an integrated quality characteristic represents. For example, the $\omega_{l}$ for PM (I) is:

$$
\begin{array}{rl}
W_{l} & =\prod_{j=1}^{m_{j}}\left(\frac{\varepsilon_{j}}{\varepsilon_{j}^{(0)}}\right)\left(\frac{v_{j}}{v_{j}^{(0)}}\right)^{2}=0.789 * 0.778 * 0.733 * \\
0.756 & * 0.800=0.272 .
\end{array}
$$

$W_{(l(1 \div 5))}=0.340$ available factors capabilities. PM (I) communication and team culture were used in separate profiles; therefore only 5 factors were satisfied.

\section{Algorithm SeLECTION}

The model describes the basis for formulating a simple algorithm for selecting the most suitable leadership of the profile that matches the project manager job position. Table II illustrates this algorithm. First, a leadership profile is constructed for each aspect. Then, a profile for each candidate manager is constructed and filtered out. Before going into any further processing, some profiles were excluded due to violation (e.g., not having the minimum number of years of experience). The value of the profile is computed and stored in a list. The top value corresponds to the top profile selected. Three alternative candidates were considered for the project management's position. The required and available leadership profiles (skills and knowledge) are defined. The most suitable project manager also needs to be selected. An evaluation technique for determining leadership capability and compatibility for managers is used to identify leadership profiles. In case of
PM (I); the knowledge/ skill compatibility weight is the highest. Experts' judgment may conclude who is a suitable candidate when the same capability weight is allocated to the project candidates. The compatibility weight and length characteristics show how a candidate capability is suitable to project needs.

\section{AN ILLUSTRATIVE EXAMPLE}

An illustrative scenario of the organisations within private and governmental agencies in the UAE are shown in Table II, outlining the capability and compatibility for each leadership characteristic, for both the experience and competence criteria. The experience ranges from 1 to 15 years while the minimum competence depends on the characteristics. In general, the level of competence ranges from 1 to 3 , where one denotes beginners, two refers to intermediate skills, and three refers to advanced skillful manager. In addition, each characteristic is associated with a special weight of significance.

In view of the presentation of this leadership profile, we need to select the best matching manager among the three alternative candidates whose profiles are shown in Table II. Using the selection algorithm, members of the candidate set are eliminated by factoring out the managers whose characteristics did not fulfil the necessary requirements in this profile. In that respect, PM (III) should be excluded because the minimum competence level constraint is not satisfied; the candidate is in level one under the DecisionMaking and Understanding Organisation's Culture characteristics for leader capability.

Therefore, the selection is confined to PM (I) and PM (II). By sorting the two profiles based on their values, we conclude that the candidate PM (I) should be selected because the value of leadership profile is higher. 


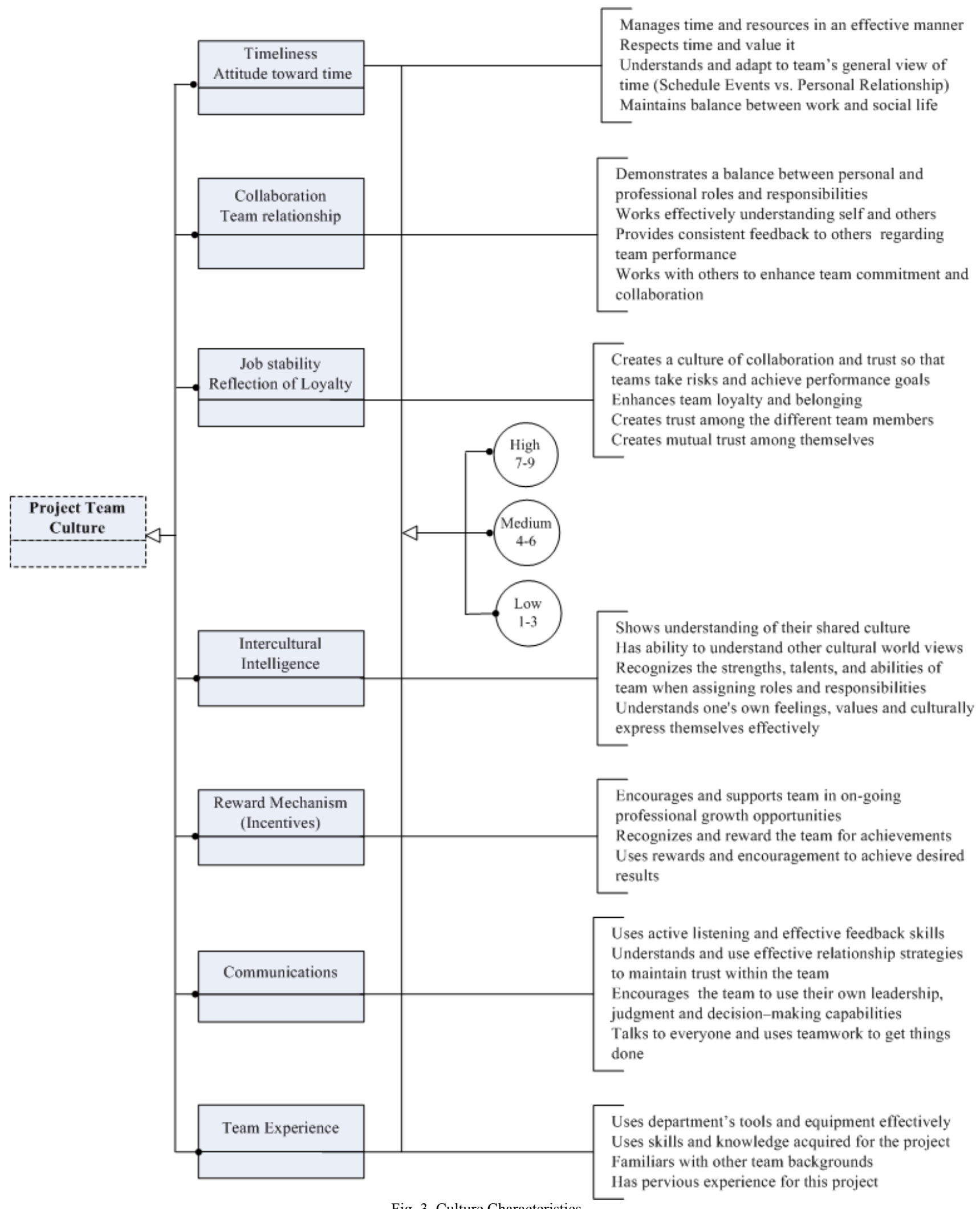

Fig. 3. Culture Characteristics

\section{CONCLUSION}

Culture and leadership play an essential role in all elements of work. The characteristics and interrelationships must be explicitly addressed for organisations to perform efficiently and successfully. Through surveys and statistical analyses, this research has identified the important cultural and leadership factors which relate to software development projects.
The results show that leadership and cultural attributes affect the effort required for the completion of a software project. The experience of the team that develops a software project along, with the interaction of the members of the team are the most important factors that affect the effort. This research can encourage other researchers to build empirical datasets in order to produce further estimation studies. 


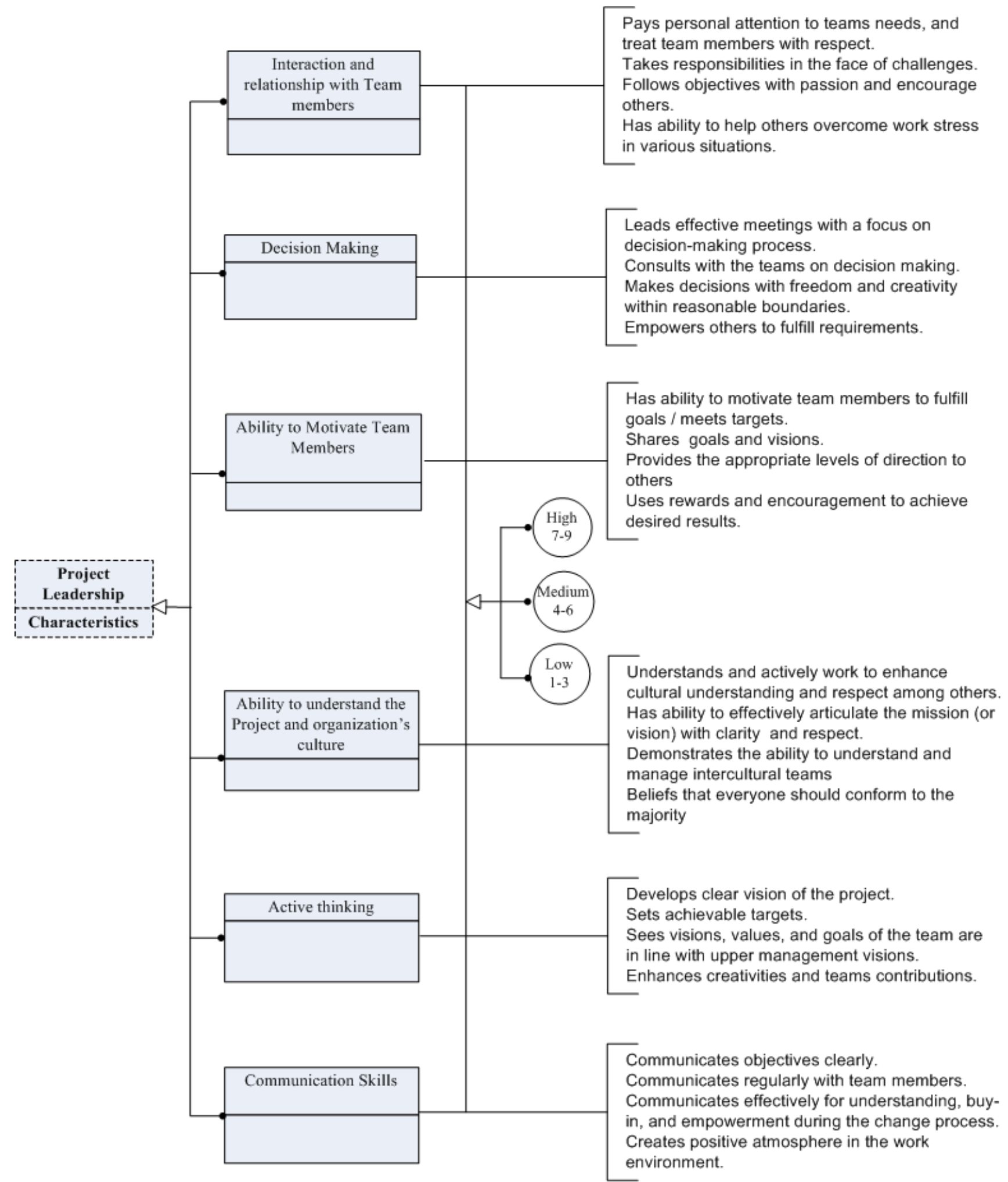

Fig. 4. Leadership Characteristics

This work introduces a profile-based approach to measurement and evaluation of leadership and cultural capabilities and compatibilities factors. This was carried out based on the premise that leadership characteristics have their influence on the performance of the project and its final total cost [6]. This method focuses on devising a formal by which a company can systematically choose the best manager in light of leadership characteristics. Formal models and an algorithm selection are presented for this purpose. The work is illustrated using an example wherein the parameters are empirically driven. This formal approach could be adapted to be part of a staff recruitment process. The prototype was developing using Excel. However, a customized software tool could be built, using the algorithms proposed in this paper.

\section{REFERENCES}

[1] W. Fairholm, Values Leadership: Toward a New Philosophy of Leadership, NY: Greenwood, 1991, pp. 4-50. 
[2] H. Schein, Organisational Culture and Leadership, 3rd edition, Joddry-Bass, John Wiley \& Sons, Inc, 2004, pp. 225.

[3] K. Hamdan, F. Abu Sitta, J. Moses, P. Smith, An Investigation into the Gulf States Government Approaches to Software Development and Effort Estimation, UK: BCS-SQM, 2005.

[4] K. Hamdan, "An investigation into software estimation methods," Ph.D dissertation, Dept. Elect and Electronic Eng., Sunderland Univ., UK, 2008.

[5] K. Hamdan, B. Belkhouche, and P. Smith, "The Influence of Culture and Leadership on Cost Estimation," in Proceedings of the International Conferences on Software Process and Product Measurement, Germany, 2008, pp.223-232.

[6] M. Shepperd and M. Jorgensen, "A Systematic Review of Software development Cost Estimation Studies," IEEE, vol. 33, no. 1, 2007.

[7] L. Angelis and I. Stamelos, "A Simulation Tool for Efficient Analogy Based Cost Estimation," Empirical Software Engineering, vol. 5, pp. 35-68, 2000 .
[8] A. Stellman, J. Greene, Applied Software Project Management, O'Reilly Media Inc, CA, 2006.

[9] V. Plekhanova, Applications of the Profile Theory to Software Engineering and Knowledge Engineering, Chicago, USA, 2000, pp. 133-141.

[10] V. Plekhanova, "On the Compatibility of Contemporary Project Management Tools with Software Project Management," Information Systems Management, Orlando, USA, 2000.

[11] S. Schneider, Jean-Louis Barsoux, Managing Across Cultures, Pearson Education- 2nd ed., England, 2003, pp. 352.

[12] R. Futrell, D. Shafer, and L. Shafer, Quality Software Project Management, Software Quality Institute, IV Series, Prentice Hall, 2002, NJ.

[13] B. Kitchenham, S. Pfleeger, and N. Fenton, "Towards a Framework for Software Validation," IEEE Trans. on S.E., vol. 21, no. 12, 1995. 